\title{
Exterior optical cloaking and illusions by using active sources: A boundary element perspective
}

\author{
H. H. Zheng, ${ }^{1}$ J. J. Xiao, ${ }^{1,2}$ Y. Lai, ${ }^{1}$ and C. T. Chan ${ }^{1}$ \\ ${ }^{1}$ Department of Physics and William Mong Institute of NanoScience and Technology, The Hong Kong University of Science and \\ Technology, Clear Water Bay, Hong Kong, China \\ ${ }^{2}$ Department of Electronic and Information Engineering and Key Laboratory of Network Oriented Intelligent Computation, \\ Shenzhen Graduate School, Harbin Institute of Technology, Shenzhen 518055, China
}

(Received 3 February 2010; revised manuscript received 28 April 2010; published 21 May 2010)

\begin{abstract}
Recently, it was demonstrated that active sources can be used to cloak any objects that lie outside the cloaking devices [F. Guevara Vasquez, G. W. Milton, and D. Onofrei, Phys. Rev. Lett. 103, 073901 (2009)]. Here, we propose that active sources can create illusion effects so that an object outside the cloaking device can be made to look like another object. Invisibility is a special case in which the concealed object is transformed to a volume of air. From a boundary element perspective, we show that active sources can create a nearly "silent" domain which can conceal any objects inside and at the same time make the whole system look like an illusion of our choice outside a virtual boundary. The boundary element method gives the fields and field gradients, which can be related to monopoles and dipoles, on continuous curves which define the boundary of the active devices. Both the cloaking and illusion effects are confirmed by numerical simulations.
\end{abstract}

DOI: 10.1103/PhysRevB.81.195116

PACS number(s): 41.20.Jb, 42.79.-e

\section{INTRODUCTION}

The classical wave-scattering cross section of an object can be significantly larger or smaller than the geometric cross section. ${ }^{1,2}$ Recipes to achieve invisibility (zero cross section) are particularly intriguing, ${ }^{3-11}$ normally enabled by the concept of transformation media and artificial metamaterials. ${ }^{6,12-14}$ The correspondence between coordinate transformation and material parameters was noted nearly half a century $\mathrm{ago}^{3}$ and such correspondence was explicitly formulated as the technique of "transform optics" to achieve invisibility by steering electromagnetic waves around a domain. ${ }^{6,7}$ Similar approaches to achieve invisibility were also proved mathematically for geometric optics ${ }^{4,5}$ and in the quasistatic limit. 8 ,9 These invisibility devices typically work by steering light around an object and the material shells need to encircle the object to be cloaked. ${ }^{6}$ It was then proposed that "cloaking at a distance" can be achieved, ${ }^{15}$ and the concept of cloaking can be extended to create arbitrary illusions. ${ }^{16}$ However, these recipes are based on artificial metamaterials and usually have bandwidth limitations. Recently, cloaking by using active sources have been proposed, ${ }^{17-20}$ which removes the requirement of metamaterials as well as the bandwidth limitation. Miller first gave a detailed prescription to perform active-source cloaking, and considered the case in which the sources encircle the cloaked domain. ${ }^{17}$ It was then shown by Vasquez et al. ${ }^{18}$ that exterior cloaking can be also realized by using several points (disks) of active multipole sources placed around the object to be cloaked. This cloaking effect has been demonstrated numerically in a broadband fashion. ${ }^{21}$

In this paper, we employ a boundary element formulation to show that arbitrary illusions can be achieved using simple active sources, i.e., sources of fields and field gradients (monopoles and dipoles), placed on continuous curves; and active-source remote cloaking is a special case. When the active sources are tuned properly according to incoming waves, a nearly "silent" domain is created such that any ob- jects can be hidden inside. At the same time, invisibility is achieved by reducing the "scattered" waves of the active sources to be almost zero on a virtual boundary which encloses the whole system. We also extend this scheme of external active cloaking to create arbitrary illusions, by tuning the active sources to produce the same scattered waves on the virtual boundary as those scattered by the object chosen for illusion under the same incident wave. The physics of the cloaking and illusion effects by active sources can be understood clearly from the boundary element perspective. ${ }^{22,23}$

\section{BOUNDARY INTEGRAL METHOD}

In this section, we demonstrate that the problem of twodimensional (2D) active cloaking can be formulated by using a boundary integral equation ${ }^{22,23}$ and be extended to create arbitrary illusions. A schematic figure of the configuration is shown in Fig. 1. The active sources are placed on boundary of the domains marked by $\Gamma_{a}$. These active sources will generate fields so that any object inside a certain domain $\Omega_{c}$ will become invisible and the external observer (outside a virtual

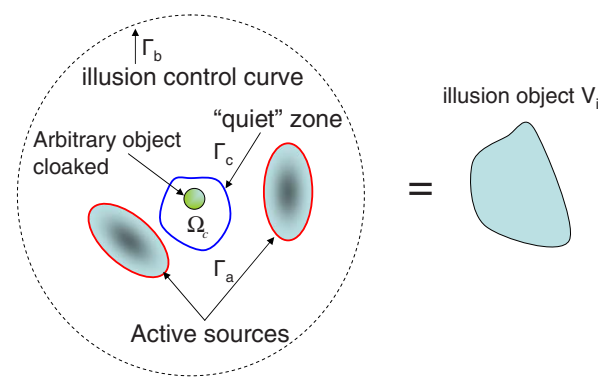

FIG. 1. (Color online) Schematic figure for the illusion devices containing exterior active sources on the boundaries $\Gamma_{a}$ that create a cloaked region $\Omega_{c}$ which can hide arbitrary scatterers inside and at the same time make the total system response like another illusion scatterer $V_{i}$ outside a virtual boundary $\Gamma_{b}$. When the illusion scatterer is just free space, invisibility is achieved. 
boundary $\Gamma_{b}$ ) will see an illusion of another object inside. The active sources do not need to encircle the object to be cloaked, as shown in Fig. 1. For any incoming waves, these active sources generate opposite fields that cancel the incoming waves inside the domain $\Omega_{c} \subset R^{2}$ to make the total fields inside $\Omega_{c}$ almost zero. Any objects inside this "quiet" zone will be concealed as the objects experience no incident wave and thus no scattering will occur.

At the same time, the active sources can be used to generate outgoing fields outside the boundary $\Gamma_{b}$ that mimic those scattered from another object $V_{i}$ under the illumination of the same incoming wave, rendering the whole system to appear like the object $V_{i}$ for any observers outside $\Gamma_{b}$. In other words, the wave fronts on $\Gamma_{b}$ of the scattering fields from the object $V_{i}$ are reconstructed by the active sources. Thus, the active sources can create an illusion so that any object placed inside $\Gamma_{c}$ is transformed optically and it looks like another object for observers outside $\Gamma_{b}$. If the fields generated by active sources cancel each other outside a virtual boundary (labeled as $\Gamma_{b}$ ), then any observer outside $\Gamma_{b}$ would not see the object inside $\Gamma_{c}$ as well as the active sources. In other words, the object inside $\Omega_{c}$ is cloaked by the active sources. In this sense, invisibility is a special case in which the illusion object $V_{i}$ is just free space.

The predesigned object $V_{i}$, which is the illusion we want to create, can be changed on demand as long as we can compute the scattered fields of this object under the incoming probing waves. In this active-source approach, the external illusion device does not need to be tailor made for the object and illusion, in contrast to the exterior cloak built with passive metamaterials, ${ }^{16}$ and there is no intrinsic bandwidth limitation. However, one needs to know in advance the probing wave or one must set up sufficiently fast-responsive sensors to capture the information of the probing wave on the boundary of $\Omega_{c}{ }^{17}$

We will formulate the problem using the boundary element approach. We note that the surface integral equation (SIE) of the 2D Helmholtz equation $\left(\nabla^{2}+k^{2}\right) \phi(\mathbf{r})=0$, where $k$ is the wave number, can be written as ${ }^{22}$

$$
\left.\phi(\mathbf{r})\right|_{\mathbf{r} \in \Omega}=\oint_{\partial \Omega} d s\left[g(\mathbf{s}, \mathbf{r}) \partial_{\mathbf{n}} \phi(\mathbf{s})-\phi(\mathbf{s}) \partial_{\mathbf{n}} g(\mathbf{s}, \mathbf{r})\right] .
$$

Here $\mathbf{n}$ is the outward unit normal vector on the boundary and $\partial_{\mathbf{n}}$ represents the normal gradient. The 2D SIE tells us that inside a homogeneous domain $\Omega$, the scalar wave function $\phi(\mathbf{r})$ is completely determined by the fields and the normal derivatives on the boundary, connected by the Green's function $g\left(\mathbf{r}, \mathbf{r}^{\prime}\right)=\frac{i}{4} H_{0}^{(1)}\left(k\left|\mathbf{r}-\mathbf{r}^{\prime}\right|\right)$, where $H_{0}^{(1)}$ is the zeroth order of the first kind of Hankel function. The counterpart of Eq. (1) for an open domain which might also involves an incident field $\phi^{\text {inc }}(\mathbf{r})$, reads $\left.\phi(\mathbf{r})\right|_{\mathbf{r} \in\left\{R^{2}-\Omega\right\}}=\phi^{\text {inc }}(\mathbf{r})+\phi^{b c}(\mathbf{r})$, where $\phi^{b c}$ is contributed from the following boundary integral:

$$
\phi^{b c}(\mathbf{r})=-\oint_{\partial \Omega} d s\left[g(\mathbf{s}, \mathbf{r}) \partial_{\mathbf{n}} \phi(\mathbf{s})-\phi(\mathbf{s}) \partial_{\mathbf{n}} g(\mathbf{s}, \mathbf{r})\right] .
$$

Inspired by Eq. (2), one can construct additional fields $\left(\phi^{b c}\right)$ in the domain $R^{2}-\Omega$, by appropriately choosing active sources to create $\phi(\mathbf{s})$ and $\partial_{\mathbf{n}} \phi(\mathbf{s})$ on the boundary $\partial \Omega$. In Appendix A, we show that such kinds of active fields can be generated by a variety of sources either located on the boundary or inside the boundary and these fields correspond to outgoing multipole radiation fields from a perspective of an observer outside the cloaking devices. Using Eq. (2), one can determine each component of the multipole sources. Now, the issue is that whether it is possible to use such kind of active sources to construct fields which can cancel the incident field $\phi^{\text {inc }}$ inside the quiet zone $\Omega_{c}$, and simultaneously mimic the scattered field in the region outside $\Gamma_{b}$ (i.e., $R^{2}-\Omega_{b}$ ), i.e.,

$$
\phi^{b c}(\mathbf{r})= \begin{cases}-\phi^{\mathrm{inc}}(\mathbf{r}) & \forall \mathbf{r} \in \Omega_{c}, \\ \phi^{\mathrm{sc}}(\mathbf{r}) & \forall \mathbf{r} \in \mathbb{R}^{2}-\Omega_{b},\end{cases}
$$

such that the total field is essentially zero inside $\Omega_{c}$ while outside $\Omega_{b}$, the total field mimics the superimposition of the incoming waves and the scattered wave of a predesigned illusion object. As shown in Appendix B, the perfectness of the cloaking and illusion effect depends on the number of active sources that we can afford to use. Employing a uniqueness theorem, ${ }^{24}$ the conditions in Eq. (3) can be simplified. In general, fixing the values of $\phi$ on the boundary (Dirichlet boundary condition) can already guarantee a unique solution in the enclosed domain for the 2D Helmholtz equation. Thus, the constraints in Eq. (3) can be replaced by

$$
\phi^{b c}(\mathbf{r})= \begin{cases}-\phi^{\text {inc }}(\mathbf{r}) & \text { for } \mathbf{r} \in \Gamma_{c}, \\ \phi^{\mathrm{sc}}(\mathbf{r}) & \text { for } \mathbf{r} \in \Gamma_{b} .\end{cases}
$$

In addition to the forgoing conditions, there is a selfconsistent condition of the boundary fields on $\Gamma_{a}{ }^{22}$

$$
\begin{aligned}
\frac{1}{2} \phi(\mathbf{s})= & \phi^{\text {inc }}(\mathbf{s})-\int_{\partial \Omega_{a}} d s^{\prime}\left\{g\left(\mathbf{s}^{\prime}, \mathbf{s}\right) \partial_{\mathbf{n}} \phi\left(\mathbf{s}^{\prime}\right)\right. \\
& \left.-\phi\left(\mathbf{s}^{\prime}\right) \partial_{\mathbf{n}} g\left(\mathbf{s}^{\prime}, \mathbf{s}\right)\right\}, \quad \mathbf{s}, \mathbf{s}^{\prime} \in \Gamma_{a}
\end{aligned}
$$

in which the integral is of a Cauchy principal value. This self-consistent condition comes from the continuity requirement when $\mathbf{r}$ approaches to the boundary from outside the cloaking device, i.e.,

$$
\lim _{\mathbf{r} \rightarrow \mathbf{s}} \phi(\mathbf{r})=\phi(\mathbf{s}), \quad \mathbf{r} \in R^{2}-\Omega_{a}, \quad \mathbf{s} \in \Gamma_{a} .
$$

In summary, the active fields are determined by the following integral equations:

$$
\begin{aligned}
& \frac{1}{2} \phi(\mathbf{r})+\int_{\partial \Omega_{a}}\left\{g(\mathbf{s}, \mathbf{r}) \partial_{\mathbf{n}} \phi(\mathbf{s})-\phi(\mathbf{s}) \partial_{\mathbf{n}} g(\mathbf{s}, \mathbf{r})\right\} d s \\
& =\phi^{\text {inc }}(\mathbf{r}), \quad \mathbf{r} \in \Gamma_{a}, \\
& -\int_{\partial \Omega_{a}}\left\{g(\mathbf{s}, \mathbf{r}) \partial_{\mathbf{n}} \phi(\mathbf{s})-\phi(\mathbf{s}) \partial_{\mathbf{n}} g(\mathbf{s}, \mathbf{r})\right\} d s=\phi^{\mathrm{sc}}(\mathbf{r}), \quad \mathbf{r} \in \Gamma_{b},
\end{aligned}
$$




$$
-\int_{\partial \Omega_{a}}\left\{g(\mathbf{s}, \mathbf{r}) \partial_{\mathbf{n}} \phi(\mathbf{s})-\phi(\mathbf{s}) \partial_{\mathbf{n}} g(\mathbf{s}, \mathbf{r})\right\} d s=-\phi^{\mathrm{inc}}(\mathbf{r}), \quad \mathbf{r} \in \Gamma_{c} .
$$

The solution to these integral equations can be numerically determined using the boundary element method (BEM), ${ }^{22,25}$ which is based on the SIE. The BEM approximates the surface integrals by discretizing the surface $\Gamma_{a} \equiv \partial \Omega_{a}$ into $N$ surface elements $\ell_{\alpha}$ on which the functions $\phi(\mathbf{s})$ and $\psi(\mathbf{s})$ $=\partial_{\mathbf{n}} \phi(\mathbf{s})$ are approximated as constants that represent the value of functions $\phi(\mathbf{s})$ and $\psi(\mathbf{s})$ across the entire element $\ell_{\alpha}$, respectively. In other words, a local step-function basis with regard to $\ell_{\alpha}$ is used to expand $\phi(\mathbf{s})$ and $\psi(\mathbf{s})$ over the entire surface $\partial \Omega$, with the expansion coefficients denoted as $\phi_{\alpha}$ and $\psi_{\alpha}$, where $\alpha=1,2, \ldots, N$. From the perspective of BEM, these expansion coefficients can be viewed as $2 N$ active sources to be determined, and Eq. (7) represents the conditions to determine these $2 N$ unknowns. Equation (7a) gives $N$ constraints. Besides these, one can choose $N_{c}$ sample points on $\Gamma_{c}$ and $N_{b}$ points on $\Gamma_{b}$ and then get a total of $N_{c}$ $+N_{b}+N$ constraints to determine the $2 N$ degree of freedom $\phi_{\alpha}$ and $\psi_{\alpha}$. We can then establish the following linear equations:

$$
\left[\begin{array}{ll}
\mathbf{H}_{c a} & \mathbf{G}_{c a} \\
\mathbf{H}_{b a} & \mathbf{G}_{b a} \\
\mathbf{H}_{a a}^{\text {int }} & \mathbf{G}_{a a}^{\text {int }}
\end{array}\right]\left[\begin{array}{l}
\boldsymbol{\Phi}_{a} \\
\boldsymbol{\Psi}_{a}
\end{array}\right]=\left[\begin{array}{c}
-\boldsymbol{\Phi}_{c}^{\mathrm{inc}} \\
\boldsymbol{\Phi}_{b}^{\mathrm{sc}} \\
\boldsymbol{\Phi}_{a}^{\text {inc }}
\end{array}\right],
$$

where $\mathbf{H}_{b a}, \mathbf{G}_{b a}$ and $\mathbf{H}_{c a}, \mathbf{G}_{c a}$ represent the interacting matrices relating the "source points" on $\Gamma_{a}$ to the field points on $\Gamma_{b}$ and $\Gamma_{c}$, and have elements defined as

$$
\begin{gathered}
H_{\alpha \beta}=\int_{\ell_{\beta}} \partial_{\mathbf{n}} g\left(\mathbf{s}, \mathbf{r}_{\alpha}\right) d s, \\
G_{\alpha \beta}=-\int_{\ell_{\beta}} g\left(\mathbf{s}, \mathbf{r}_{\alpha}\right) d s, \quad \mathbf{r}_{\alpha} \in \Gamma_{c} \cup \Gamma_{b}, \\
\alpha=1,2, \ldots, N_{c}+N_{b}, \quad \ell_{\beta} \subset \Gamma_{a}, \beta=1,2, \ldots, N
\end{gathered}
$$

whereas $\mathbf{H}_{a a}^{\text {int }}$ and $\mathbf{G}_{a a}^{\text {int }}$ represent the self-consistent conditions imposed on $\phi$ and $\partial_{\mathbf{n}} \phi$, with elements defined as

$$
\begin{gathered}
H_{\alpha \beta}^{\mathrm{int}}=\frac{1}{2} \delta_{\alpha \beta}-\int_{\ell_{\beta}} \partial_{\mathbf{n}} g\left(\mathbf{s}, \mathbf{r}_{\alpha}\right) d s, \\
G_{\alpha \beta}^{\mathrm{int}}=\int_{\ell_{\beta}} g\left(\mathbf{s}, \mathbf{r}_{\alpha}\right) d s, \quad \ell_{\alpha}, \ell_{\beta} \subset \Gamma_{a}, \\
\alpha, \beta=1,2, \ldots, N, \quad \mathbf{r}_{\alpha} \text { is the center of } \ell_{\alpha} .
\end{gathered}
$$

On the right-hand side of Eq. (8), $\boldsymbol{\Phi}_{c}^{\text {inc }}$ denotes the incoming probing wave fields $\phi^{\text {inc }}$ sampled at the inner quite zone boundary $\Gamma_{c} \equiv \partial \Omega_{c}, \boldsymbol{\Phi}_{b}^{\text {sc }}$ denotes the sampled scattered fields on the outer boundary $\Gamma_{b} \equiv \partial \Omega_{b}$ that would have been scattered by the object $V_{i}$ under the illumination of the same probing wave $\phi^{\text {inc }}$. $\boldsymbol{\Phi}_{a}^{\text {inc }}$ denotes the incoming wave fields on the boundary of the cloaking devices. Thus, the fields outside $\Gamma_{b}$ (the dashed line in Fig. 1) approach those of $\phi^{\text {inc }}$ scattered by the object $V_{i}$ as the number of sampling points $(N)$ increases and the discrepancy decreases if $N$ increases. If we set $\boldsymbol{\Phi}_{b}^{\mathrm{sc}}=0$, we achieve the active external invisible cloaking. Cloaking is thus a special case of illusion in this formulation. $\boldsymbol{\Phi}_{a}$ and $\boldsymbol{\Psi}_{a}$ are both $N$-dimensional vectors representing the total field and field gradient on $\Gamma_{a}$, which require active sources to generate. For simplicity, in our numerical calculations, we set $N_{c}+N_{b}+N=2 N$ so that the matrix in Eq. (8) is a square matrix. The linear system of equations is solved using the LAPACK subroutine ZGESV. We can see that BEM offers a physically transparent way of deriving the active sources needed to do remote cloaking and illusion and offers a straightforward numerical recipe in calculating those sources.

The forgoing discussions are restricted to nonradiating objects. If the object inside $\Omega_{c}$ itself is a radiating source, we should add extra active sources to cancel the radiated field $\phi^{\text {radiate }}$ outside $\Gamma_{b}$. This extra term is determined by

$$
\begin{gathered}
\frac{1}{2} \phi^{\text {extra }}(\mathbf{r})+\int_{\partial \Omega_{a}}\left\{\widetilde{g}(\mathbf{s}, \mathbf{r}) \partial_{\mathbf{n}} \phi^{\text {extra }}(\mathbf{s})-\phi^{\text {extra }}(\mathbf{s}) \partial_{\mathbf{n}} \widetilde{g}(\mathbf{s}, \mathbf{r})\right\} d s \\
=\phi^{\text {radiate }}(\mathbf{r}), \quad \mathbf{r} \in \Gamma_{a}, \\
-\int_{\partial \Omega_{a}}\left\{\widetilde{g}(\mathbf{s}, \mathbf{r}) \partial_{\mathbf{n}} \phi^{\text {extra }}(\mathbf{s})-\phi^{\text {extra }}(\mathbf{s}) \partial_{\mathbf{n}} \widetilde{g}(\mathbf{s}, \mathbf{r})\right\} d s \\
=-\phi^{\text {radiate }}(\mathbf{r}), \quad \mathbf{r} \in \Gamma_{b}, \\
-\int_{\partial \Omega_{a}}\left\{\widetilde{g}(\mathbf{s}, \mathbf{r}) \partial_{\mathbf{n}} \phi^{\text {extra }}(\mathbf{s})-\phi^{\text {extra }}(\mathbf{s}) \partial_{\mathbf{n}} \widetilde{g}(\mathbf{s}, \mathbf{r})\right\} d s=0, \quad \mathbf{r} \in \Gamma_{c} .
\end{gathered}
$$

The Green's function $\widetilde{g}$ might be different since the radiating field may be of a different frequency $\widetilde{\omega}$. Then the total active field should be

$$
\phi_{a}(\mathbf{r}, t)=\phi(\mathbf{r}) \exp [-i \omega t]+\phi^{\text {extra }}(\mathbf{r}) \exp [-i \widetilde{\omega} t],
$$

where $\phi(\mathbf{r})$ is the solution of Eq. (7) and $\phi^{\text {extra }}$ is the solution of Eq. (11). We note that the condition in Eq. (11c) is necessary since the radiating object may also be a passive scatterer.

In fact, in our formalism, we have assumed that the active sources (placed on $\Gamma_{a}$ ) themselves do not scatter waves. However, in real world, the sources may also scatter external fields. In this case, we can adjust the active fields generated by the active sources to cancel the scattering fields of their own on $\Gamma_{c}$ and $\Gamma_{b}$ so that the scattering effect of the active sources can be removed or minimized. To take the scattering of sources into account, in Eq. (8), we should change $-\boldsymbol{\Phi}_{c}^{\text {inc }}$ to $-\boldsymbol{\Phi}_{c}^{\mathrm{inc}}-\boldsymbol{\Phi}_{\mathrm{sr}, c}^{\mathrm{sc}}$ and $\boldsymbol{\Phi}_{b}^{\mathrm{sc}}$ to $\boldsymbol{\Phi}_{b}^{\mathrm{sc}}-\boldsymbol{\Phi}_{\mathrm{sr}, b}^{\mathrm{sc}}$, where $\boldsymbol{\Phi}_{\mathrm{sr}, c}^{\mathrm{sc}}$ and $\boldsymbol{\Phi}_{\mathrm{sr}, b}^{\mathrm{sc}}$ denote the sources scattered fields on $\Gamma_{c}$ and $\Gamma_{b}$, respectively. In general, the scattering fields of the sources $\left(\boldsymbol{\Phi}_{\mathrm{sr}, c}^{\mathrm{sc}}\right.$ and $\left.\boldsymbol{\Phi}_{\mathrm{sr}, b}^{\mathrm{sc}}\right)$ depend on the incident wave $\boldsymbol{\Phi}^{\mathrm{inc}}$ and the active fields $\left(\boldsymbol{\Phi}_{a}\right.$ and $\left.\boldsymbol{\Psi}_{a}\right)$ generated by the sources. This dependence is known as the shape and material makeup of the active sources are all known. Therefore, we can reformulate Eq. (8) 

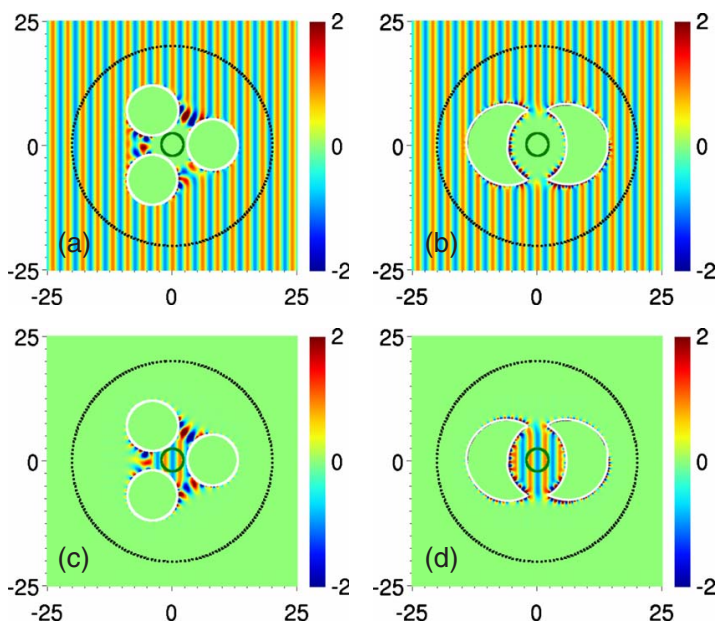

FIG. 2. (Color online) Example of active-source external cloak. (a) and (b) show the total fields and (c) and (d) show the scattered fields. The sources are arranged on the boundaries (white solid line) of three circles in the left panels while are in two crescent-shaped curves in the right panels. Here, the incoming plane wave is of wavelength $\lambda=3.0$.

by taking into account these considerations. The active fields for this case can be obtained by solving the modified linear equations.

\section{NUMERICAL SIMULATIONS}

\section{A. Remote cloaking effect}

Here, we show numerically that external cloaking is achieved by simply setting $\boldsymbol{\Phi}_{b}^{\mathrm{sc}}=0$ in Eq. (8). The choice of plane wave $\exp (i \mathbf{k} \cdot \mathbf{r})$ as the incoming source is just for simplicity but the formulation works for other forms of incident wave. The configurations of the cloaking devices are shown in Fig. 2 for two kinds of source arrangement. In the left panels, active sources are placed on three circles arranged as shown in Figs. 2(a) and 2(c). In the right panels, we show a case in which the active sources are placed on two crescentshaped curves [Figs. 2(b) and 2(d)]. In both cases, we have chosen $N=900, N_{c}=300$, and $N_{b}=600$. Employing the scheme described in the preceding section, we can achieve an approximate solution numerically. We see from Fig. 2 that the field inside the quiet zone is essentially zero and here is no scattering. The numerical solutions only ensure that Eq. (7) is correct in a finite number of points. To quantify the overall quality of the solution, we consider the following error functions (measured with $L^{2}$ norm) defined on the two circles $\Gamma_{b}(r=20)$ and $\Gamma_{c}(r=2)$ and inside the quiet zone $\Omega_{c}$,

$$
\operatorname{Err}\left(\Gamma_{b}\right)=\frac{\oint_{\partial \Omega_{b}}\left|\phi(\mathbf{s})-\phi^{\mathrm{inc}}(\mathbf{s})\right|^{2} d s}{\oint_{\partial \Omega_{b}}\left|\phi^{\mathrm{inc}}(\mathbf{s})\right|^{2} d s},
$$

$$
\begin{gathered}
\operatorname{Err}\left(\Gamma_{c}\right)=\frac{\oint_{\partial \Omega_{c}}|\phi(\mathbf{s})|^{2} d s}{\oint_{\partial \Omega_{c}}\left|\phi^{\mathrm{inc}}(\mathbf{s})\right|^{2} d s}, \\
\operatorname{Err}\left(\Omega_{c}\right)=\frac{\iint_{\Omega_{c}}|\phi(\mathbf{r})|^{2} d A}{\iint_{\Omega_{c}}\left|\phi^{\mathrm{inc}}(\mathbf{r})\right|^{2} d A} .
\end{gathered}
$$

Each integrand is numerically evaluated at a set of 40000 points sampled in the corresponding integration domain. For fixed circle sizes of $\Gamma_{b}$ and $\Gamma_{c}$, the errors depend on the choice of $N, N_{b}$, and $N_{c}$, as well as the frequency of the incoming wave. A detailed discussion on the dependence of these errors on the parameters can be found in Appendix B. It is seen that the error decreases as we increase $N$. In other words, we are able to achieve better cloaking effects if we can control the boundary fields more precisely. The field patterns are presented in Fig. 2. Figures 2(a) and 2(b) show the total fields which are the superposition of the incoming plane wave and the active fields generated by the "active sources" [solutions to Eq. (8)] placed on the white solid lines. The total fields $\phi(\mathbf{r})$ outside $\Gamma_{b}$ (marked by the black dashed line), a circle of $r=20$ units, are almost the same as the incoming plane wave, with a discrepancy $\operatorname{Err}\left(\Gamma_{b}\right)=4.62$ $\times 10^{-13}$ in Figs. 2(a) and 2(c). At the same time, we achieve a quiet zone $\Omega_{c}$ (bounded by $\Gamma_{c}$ as marked by the green thick-solid lines) within a circle of $r=2$ inside which the total fields almost vanish, with $\operatorname{Err}\left(\Gamma_{c}\right)=1.14 \times 10^{-12}$ and $\operatorname{Err}\left(\Omega_{c}\right)=1.03 \times 10^{-12}$. Figures $2(\mathrm{c})$ and $2(\mathrm{~d})$ show the corresponding scattered fields $\phi^{\mathrm{sc}}(\mathbf{r})=\phi(\mathbf{r})-\phi^{\text {inc }}(\mathbf{r})$, which are exactly the fields created by the active devices. Concomitantly, $\phi^{\mathrm{sc}}(\mathbf{r})$ vanishes outside $\Gamma_{b}$ and is the reverse of $\phi^{\text {inc }}(\mathbf{r})$ inside the quiet zone $\Omega_{c}$. The strength of the fields on the boundary $\Gamma_{a}$ is on the order of 100 [e.g., 160 in Figs. 2(a) and 2 (c) and $\sim 80$ in Figs. 2(b) and 2(d)], and can be easily achieved physically. Vasquez and co-workers ${ }^{18}$ proposed that three disjoint circular disks are needed to perform remote active cloaking. Here, we see that from Figs. 2(b) and 2(d) that a noncircular cloaking device comprising two simply connected regions can also achieve the similar cloaking effect. It is further shown (figure not presented here) that active sources on one simply connected cloaking device can also achieve a high degree of invisibility.

\section{B. Illusion effect}

Next, we demonstrate an illusion effect such that whatever objects placed inside the quiet zone $\Omega_{c}$ will appear like another object, which is chosen here to be a banana-shaped dielectric object with refractive index $n=2.32$. Figures $3(\mathrm{c})$ and 3(f) show the total and scattered fields of such a "banana" under the illumination of a plane wave $\exp (i \mathbf{k} \cdot \mathbf{r})$. We set the control boundary $\Gamma_{b}$ (marked as the dashed line) as a circle with radius $r=20$ outside which the illusion shall be observed. This requires one to set $\boldsymbol{\Phi}_{b}^{\mathrm{sc}}$ in Eq. (8) as the scat- 

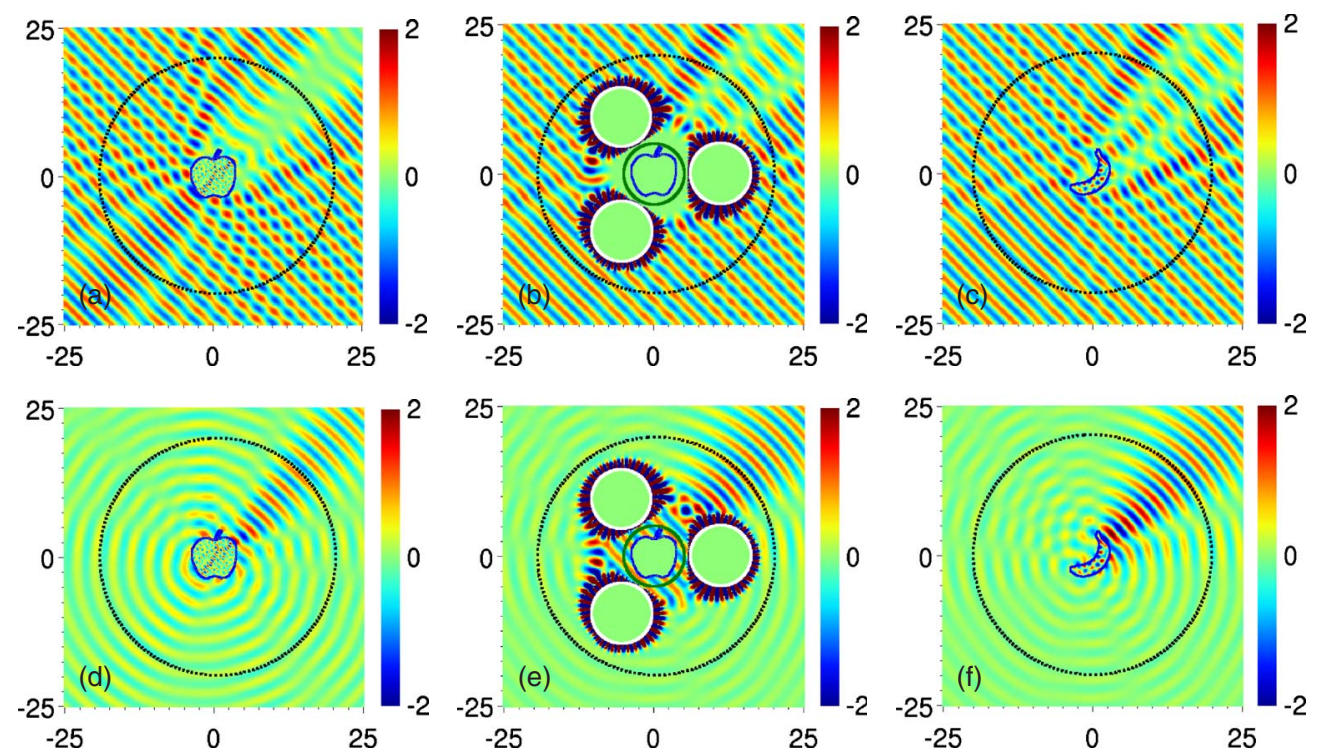

FIG. 3. (Color online) Optical illusion effect by active sources. (a), (b), and (c) show the total fields. (d), (e), and (f) show the scattered fields $\left(\phi^{\text {tot }}-\phi^{\text {inc }}\right)$. (a) and (d) are for an apple $(\epsilon=16.0, \mu=1.0)$. (b) and (e) are for an illusion device with an apple-shaped object [identical to (a) and (d)] concealed inside $\Omega_{c}$. The active sources are placed on the three circles (white solid line). (c) and (f) are for a banana-shaped dielectric object $(\epsilon=5.0, \quad \mu=1.0)$. The active sources give the same scattering pattern as the banana outside the black dotted curve.

tered fields due to the predesigned banana [see Fig. 3(c)] on $\Gamma_{b}$. A total of $N=900$ sample points (with $N_{b}=600, N_{c}$ $=300)$ are used here in the numerical calculation, and $\boldsymbol{\Phi}_{c}^{\text {inc }}$ in Eq. (8) is the function $\exp (i \mathbf{k} \cdot \mathbf{r})$ sampled over a circle of $r$ $=5$ (the boundary of the quiet zone $\Omega_{c}$ as marked by the green solid circle). In Figs. 3(b) and 3(e), we conceal an apple-shaped dielectric object $(n=4)$ inside the quiet zone. Figures 3(a) and 3(d) show the total and scattered fields of the "apple" under the illumination of the plane wave. We see in Figs. 3(b) and 3(e) that, after turning on the active sources, the total system responses to the incoming plane wave in a way as if a banana is placed in the cloaked region [Figs. 3(c) and $3(\mathrm{f})]$. The field discrepancy on the circle of $r=20$ is $1.17 \times 10^{-6}$, measured with $L^{2}$ norm as,

$$
\operatorname{Err}^{i l l}\left(\Gamma_{b}\right)=\frac{\oint_{\partial \Omega_{b}}\left|\phi(\mathbf{s})-\phi^{\mathrm{inc}}(\mathbf{s})-\widetilde{\phi}^{\mathrm{sc}}(\mathbf{s})\right|^{2} d s}{\oint_{\partial \Omega_{b}}\left|\phi^{\mathrm{inc}}(\mathbf{s})\right|^{2} d s},
$$

where $\widetilde{\phi}^{\text {sc }}$ is the scattered field of the predesigned banana under the illumination of the incoming wave. Figure 3(e) shows that the active sources construct a negative counterpart of the incoming plane wave to create the quiet zone $\Omega_{c}$ so that the total field surrounding the apple is almost zero. We note that we can put any nonradiating object inside $\Omega_{c}$ without affecting the total scattering pattern outside $\Gamma_{b}$ [see Figs. 4(b) and 4(c)] since all the fields inside $\Omega_{c}$ are almost zero as is shown in Fig. 4(a). [The discrepancies: $\operatorname{Err}^{i l l}\left(\Gamma_{c}\right)$ $=6.15 \times 10^{-7}, \operatorname{Err}^{i l l}\left(\Omega_{c}\right)=1.11 \times 10^{-6}$.] In fact, any passive object inside $\Omega_{c}$ does not "talk" to the other parts of the world, rendering this illusion device workable for multiple and arbitrary objects. In principle, by changing the active sources, we can let observers outside $\Gamma_{b}$ see whatever we want them to see.

\section{Cloaking a radiating object}

In this section, we demonstrate the cloaking effect of a radiating object. For simplicity, we assume that $\phi^{\text {radiate }}$ $=10 \cdot H_{1}^{(1)}(\tilde{k} r) \cos \theta \exp [-i \widetilde{\omega} t]$ and there is no incoming wave from the outside. The simulation results are shown in Fig. 5, with $\tilde{k}=\pi$. Here, we use the same parameters $N, N_{b}, N_{c}$ as those in Secs. III A and III B. From Fig. 5(b), we see that the total field pattern near the dipole remain the same with Fig. 5(a). The cloaking device creates active fields which cancel the radiating fields outside $\Gamma_{b}$ [see Figs. 5(b) and 5(c)] and do not affect the field pattern inside $\Omega_{c}$. The active source in this example is just the $\phi^{\text {extra }}(\mathbf{s})$ which is the solution of Eq. (11). Considering the linear superposition property, one can add together the solution of Eq. (7) and $\phi^{\text {extra }}$ to achieve cloaking or illusion effects for this specific radiating object.

\section{CONCLUSION}

In conclusion, we apply a boundary element method to demonstrate both external cloaking and illusion effects using active sources on continuous curves for the 2D Helmholtz equation. The scalar wave formalism applies to both acoustic waves and electromagnetic waves in two dimensions. This approach works for arbitrary objects and there is no intrinsic bandwidth limitation. The limitation of this type of remote active cloaking and illusion is that it requires the prior knowledge of the incoming wave or the availability of sensors that can detect the fields quickly enough on the boundaries and of active sources that can respond fast enough. ${ }^{17}$

\section{ACKNOWLEDGMENTS}

This work was supported by Hong Kong RGC under 

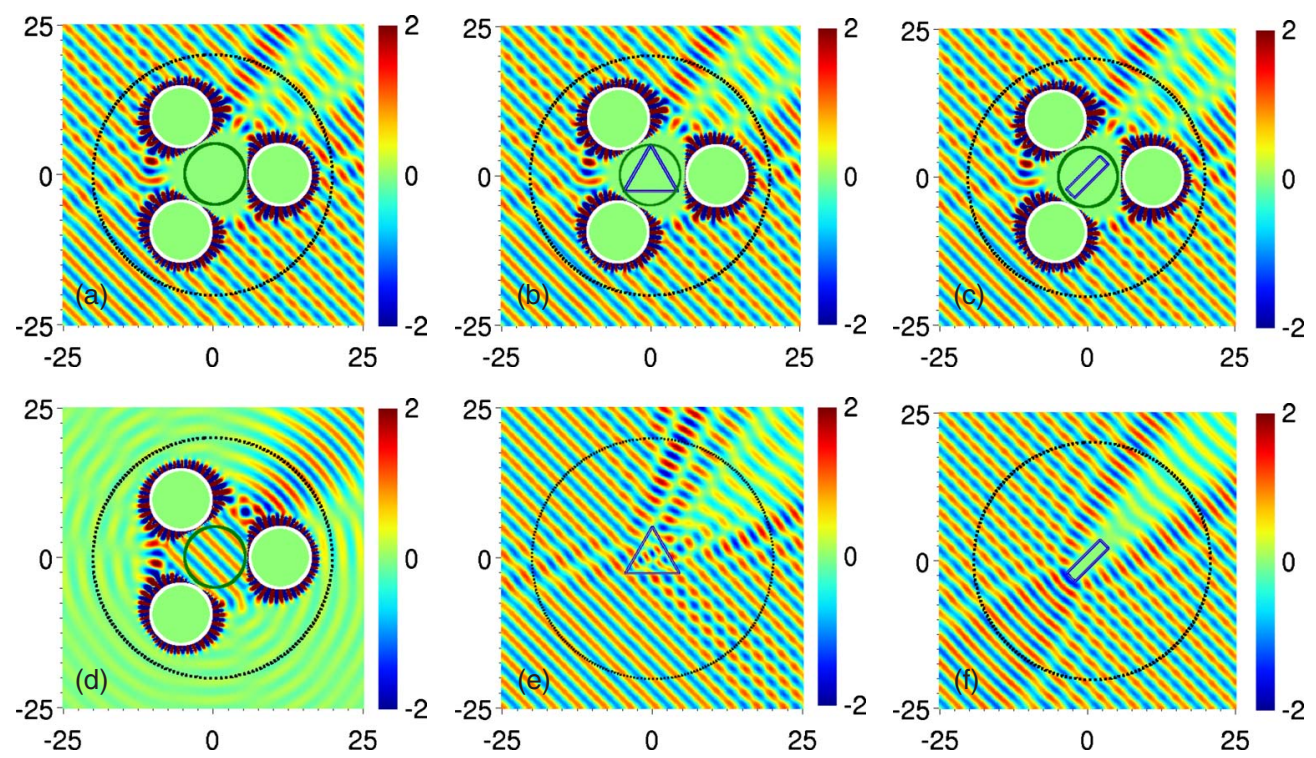

FIG. 4. (Color online) Optical illusion effect by active sources. (a) shows the total field pattern of an illusion device, and (d) shows the pattern of the active field generated by the illusion device. (b) and (c) are patterns of the total fields for the same illusion device but with different objects concealed inside $\Omega_{c}$ [(b) is a triangle $\epsilon=2.25, \mu=-1.0$ and (c) is a PEC slab]. (e) and (f) are patterns of the total fields for the objects identical to those concealed inside the quiet zones in (b) and (c). Any object can be concealed inside the quiet zone where the total fields are almost zero.

Grant No. 600209. Computation resources are supported by Shun Hing Education and Charity Fund. We acknowledge discussions with Z. H. Hang and Jeffery Lee.

\section{APPENDIX A: POSSIBLE WAYS TO BUILD UP THE ACTIVE SOURCES}

The BEM gives the field and field gradients on the boundary of the active cloaking device. It does not give directly the sources that give rise to those fields. There are obviously many different ways of arranging sources within the boundary of the active devices to generate the necessary fields. In this appendix, we give a few examples.

\section{Using monopole sources and dipolar sources located on the boundaries}

We note that the active fields are given by

$$
\phi^{\text {active }}(\mathbf{r})=-\oint_{\partial \Omega_{a}} d s\left[g(\mathbf{s}, \mathbf{r}) \partial_{\mathbf{n}} \phi_{a}(\mathbf{s})-\phi_{a}(\mathbf{s}) \partial_{\mathbf{n}} g(\mathbf{s}, \mathbf{r})\right]
$$

where $\phi_{a}$ and $\partial_{\mathbf{n}} \phi_{a}$ are the solutions of Eq. (7). The first term represents a set of monopole point sources, with the corresponding strengths as follows:

$$
p_{i}=-\partial_{\mathbf{n}} \phi_{a}\left(\mathbf{s}_{i}\right) \delta a_{i},
$$

where $\mathbf{s}_{i}$ is the center of the $i$ th boundary element and $\delta a_{i}$ is the length of the corresponding element. The second term represents a set of dipole sources on the boundary since

$$
\begin{aligned}
\partial_{\mathbf{n}} g(\mathbf{s}, \mathbf{r})= & \frac{i k}{4} H_{1}^{(1)}(k|\mathbf{r}-\mathbf{s}|) \frac{\mathbf{r}-\mathbf{s}}{|\mathbf{r}-\mathbf{s}|} \cdot \mathbf{n}=\frac{i k e^{i \theta^{\prime}}}{8} H_{1}^{(1)}(k|\mathbf{r}-\mathbf{s}|) e^{i \theta} \\
& +\frac{i k e^{-i \theta^{\prime}}}{8} H_{1}^{(1)}(k|\mathbf{r}-\mathbf{s}|) e^{-i \theta},
\end{aligned}
$$

which are exactly dipole sources located at $s_{i}$. Here, $\theta^{\prime}$ is the
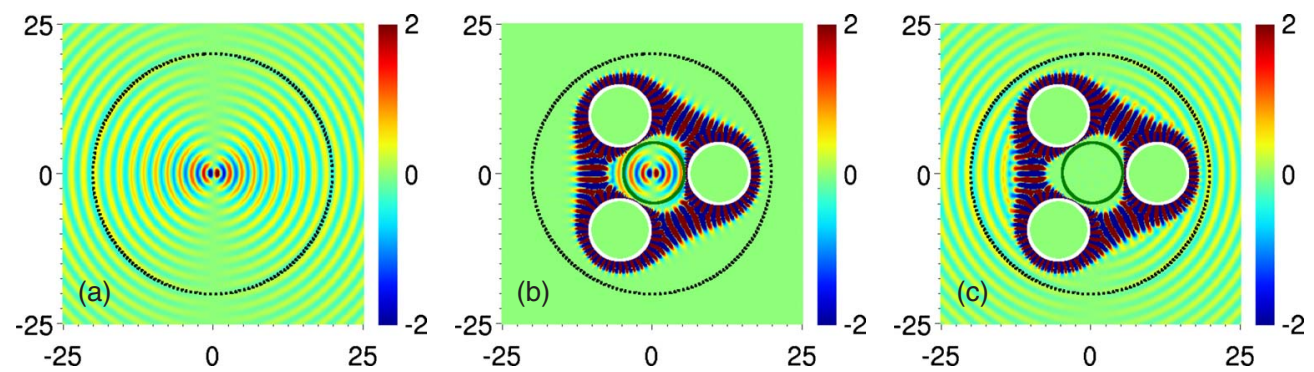

FIG. 5. (Color online) Cloaking a radiating object. (a) The radiating field pattern of $10 \cdot H_{1}^{(1)}(\tilde{k} r) \cos \theta \exp [-i \widetilde{\omega} t]$, where $\tilde{k}=\pi$. (b) The total field pattern with the sources on the boundary of the cloaking device actives (three circles) radiating according to the solutions of Eq. (11). (c) The field generated by the cloaking device. 


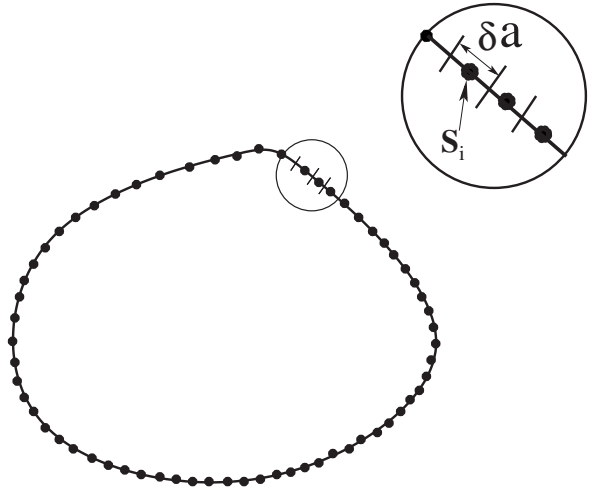

FIG. 6. Generating active field by using monopole sources and dipolar sources located on the boundaries of the cloaking or illusion devices.

direction of the unit normal $\mathbf{n}$ and $\theta$ is the direction of $\mathbf{r}-\mathbf{s}$. We have used the recursion relation of the Hankel functions $\frac{d H_{0}^{(1)}(x)}{d x}=-H_{1}^{(1)}(x)$.

The corresponding strengths of the two types of dipole sources are

$$
\begin{gathered}
q_{i_{1}}=\frac{i k e^{i \theta_{i}^{\prime}} \delta a_{i}}{8} \phi_{a}\left(\mathbf{s}_{i}\right), \quad \text { for } H_{1}^{(1)}\left(k\left|\mathbf{r}-\mathbf{s}_{i}\right|\right) e^{i \theta}, \\
q_{i_{2}}=\frac{i k e^{-i \theta_{i}^{\prime}} \delta a_{i}}{8} \phi_{a}\left(\mathbf{s}_{i}\right), \text { for } H_{1}^{(1)}\left(k\left|\mathbf{r}-\mathbf{s}_{i}\right|\right) e^{-i \theta} .
\end{gathered}
$$

It can be easily verified that this set of point sources and dipole sources can exactly generate the necessary active fields (see the schematic configuration in Fig. 6).

\section{Using double layers of point sources}

We can also use only point sources to build up the same necessary active fields. ${ }^{17}$ Instead of positioning the sources on the boundary $\Gamma_{a}$, one can place point sources on the outer boundary $S_{\text {out }}$ and the inner boundary $S_{\text {in }}$ (see the schematic configuration in Fig. 7), i.e., we place point sources at the following points:

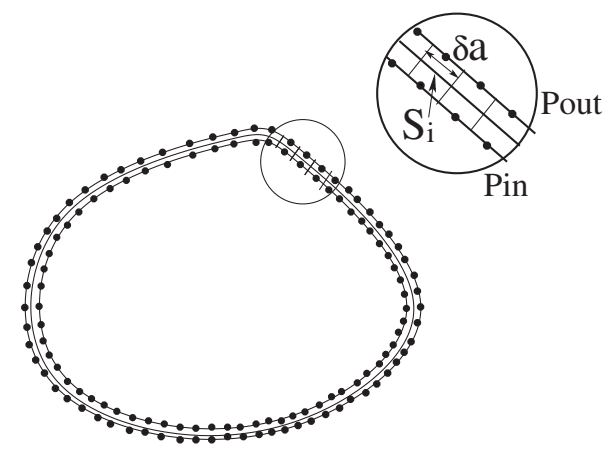

FIG. 7. Generating active field by using point sources located on double-layer boundaries.

$$
\mathbf{s}_{i}^{\text {out }}=\mathbf{s}_{i}+\frac{h}{2} \mathbf{n}_{i}, \quad \mathbf{s}_{i}^{\text {in }}=\mathbf{s}_{i}-\frac{h}{2} \mathbf{n}_{i}, \quad i=1,2, \ldots, N,
$$

where $\mathbf{s}_{i}$ is the center of the $i$ th element and $\mathbf{n}_{i}$ is the unit normal of the $i$ th element. The corresponding strengths of the point sources are

$$
\begin{aligned}
& p\left(\mathbf{s}_{i}^{\text {out }}\right)=\frac{\delta a_{i}}{h}\left[\phi_{a}\left(\mathbf{s}_{i}\right)-\frac{h}{2} \partial_{\mathbf{n}} \phi_{a}\left(\mathbf{s}_{i}\right)\right], \\
& p\left(\mathbf{s}_{i}^{\text {in }}\right)=-\frac{\delta a_{i}}{h}\left[\phi_{a}\left(\mathbf{s}_{i}\right)+\frac{h}{2} \partial_{\mathbf{n}} \phi_{a}\left(\mathbf{s}_{i}\right)\right] .
\end{aligned}
$$

It can easily seen that the field generated by the point sources above is

$$
\begin{aligned}
\phi(\mathbf{r})= & \sum_{i}^{N}\left[p\left(\mathbf{s}_{i}^{\text {out }}\right) g\left(\mathbf{s}_{i}^{\text {out }}, \mathbf{r}\right)+p\left(\mathbf{s}_{i}^{i n}\right) g\left(\mathbf{s}_{i}^{i n}, \mathbf{r}\right)\right]= \\
& -\oint_{\partial \Omega_{a}} d s\left[g(\mathbf{s}, \mathbf{r}) \partial_{\mathbf{n}} \phi_{a}(\mathbf{s})-\phi_{a}(\mathbf{s}) \partial_{\mathbf{n}} g(\mathbf{s}, \mathbf{r})\right]+O\left(h^{2}\right) .
\end{aligned}
$$

As long as the distance $h$ between the outer and the inner boundary is sufficiently small, one can build up the active field as close as we like to that of Eq. (A1).

\section{Using multipole sources placed at the centers of the cloaking or illusion devices}

Instead of positioning monopole or dipole sources directly on the boundaries of the cloaking or illusion devices, one can put multipole active sources inside the cloaking devices $\Omega_{a}$ to construct the required boundary fields $\phi$ and $\partial_{\mathbf{n}} \phi$, and then control the fields outside $\Omega_{a}$. For a circular domain, it is convenient to place the multipole source at the center. In the following, we will discuss a way to determine the corresponding strength of each component from the boundary fields obtained by BEM.

We note that the active fields are given by

$$
\phi^{\text {active }}(\mathbf{r})=-\oint_{\partial \Omega_{a}} d s\left[g(\mathbf{s}, \mathbf{r}) \partial_{\mathbf{n}} \phi_{a}(\mathbf{s})-\phi_{a}(\mathbf{s}) \partial_{\mathbf{n}} g(\mathbf{s}, \mathbf{r})\right] .
$$

For the domain outside the cloaking device, $\left|\mathbf{s}-\mathbf{r}_{c}\right|<\left|\mathbf{r}-\mathbf{r}_{c}\right|$ ( $\mathbf{r}_{c}$ is the center of the cloaking device), we can expand the Green's function as

$$
\begin{gathered}
g(\mathbf{s}, \mathbf{r})=\frac{i}{4} \sum_{m=-\infty}^{\infty} H_{m}^{(1)}\left(k\left|\mathbf{r}-\mathbf{r}_{c}\right|\right) J_{m}\left(k\left|\mathbf{s}-\mathbf{r}_{c}\right|\right) e^{i m \Delta \theta}, \\
\left|\mathbf{s}-\mathbf{r}_{c}\right|<\left|\mathbf{r}-\mathbf{r}_{c}\right|,
\end{gathered}
$$

where $H_{m}^{(1)}$ is the $m$ th-order Hankel function of the first type and $\Delta \theta$ is the angle between $\mathbf{r}-\mathbf{r}_{c}$ and $\mathbf{s}-\mathbf{r}_{c}$. Substituting this into Eq. (A8), one can get the corresponding multipole sources. For example, for the case shown in Fig. 2(a), one can use multipole sources locating at the centers of the three 
circles $r_{c_{j}}, j=1,2,3$ to generate the required fields and field gradients, i.e.,

$$
\phi^{\text {active }}(\mathbf{r})=\sum_{j=1}^{3} \sum_{m=-\infty}^{\infty} a_{m, j} H_{m}^{(1)}\left(k\left|\mathbf{r}-\mathbf{r}_{c_{j}}\right|\right) e^{i m \theta},
$$

where $\theta$ is the direction of $\mathbf{r}-\mathbf{r}_{c_{j}}$ and $a_{m, j}$ is the strength of the multipole source $H_{m}^{(1)}$ locating at $r_{c_{j}}$, which can be obtained by the following integrals:

$$
\begin{aligned}
a_{m, j}= & \frac{i}{4} \oint_{\partial \Omega_{a_{j}}} d s e^{-i m \theta^{\prime}}\left\{\partial_{\mathbf{n}} J_{m}\left(k \mid \mathbf{s}-\mathbf{r}_{c_{j}}\right) \phi_{a}(\mathbf{s})-J_{m}(k \mid \mathbf{s}\right. \\
& \left.\left.-\mathbf{r}_{c_{j}} \mid\right) \partial_{\mathbf{n}} \phi_{a}(\mathbf{s})\right\},
\end{aligned}
$$

where $j=1,2,3, J_{m}(x)$ is the $m$ th-order Bessel function and $\theta^{\prime}$ is the direction of $\mathbf{s}-\mathbf{r}_{c_{j}}$.

The multipole expansion of the Green's function demonstrates that the two kinds of boundary fields and their gradients [that is, $\phi(\mathbf{s})$ and $\left.\partial_{\mathbf{n}} \phi(\mathbf{s})\right]$ contribute the same form of multipole radiation fields $H_{m}(k r) \exp (\operatorname{im} \theta)$, with their strengths determined by the surface integral. In this sense, $\phi(\mathbf{s})$ and $\partial_{\mathbf{n}} \phi(\mathbf{s})$ are on the same footing in generating the active field in the open domain (i.e., $R^{2}-\Omega_{a}$ ). This indicates that, in Eq. (A8), there are multiple choices of the boundary fields $\phi_{a}$ and $\partial_{\mathbf{n}} \phi_{a}$, for the same $\phi^{\text {active }}(\mathbf{r})$. This is reasonable, as one can easily verify that

$$
\begin{aligned}
& \oint_{\partial \Omega_{a}} d s\left[\phi^{k}(\mathbf{s}) \partial_{\mathbf{n}} g(\mathbf{s}, \mathbf{r})-g(\mathbf{s}, \mathbf{r}) \partial_{\mathbf{n}} \phi^{k}(\mathbf{s})\right]=0, \\
& \quad \text { for } \mathbf{r} \in R^{2}-\Omega_{a},
\end{aligned}
$$

if $\phi^{k}(\mathbf{r})$ satisfies the Helmholtz equation

$$
\left(\nabla^{2}+k^{2}\right) \phi^{k}(\mathbf{r})=0, \quad \mathbf{r} \in \Omega_{a} .
$$

If a $\left\{\phi_{a}(\mathbf{s}), \partial_{\mathbf{n}} \phi_{a}(\mathbf{s})\right\}$ pair can give the required active sources, any solution of the form $\left\{\phi_{a}(\mathbf{s})+\phi^{k}(\mathbf{s}), \partial_{\mathbf{n}} \phi_{a}(\mathbf{s})+\partial_{\mathbf{n}} \phi^{k}(\mathbf{s})\right\}$ gives the same active fields. Then a question arises: Is the solution obtained by Eq. (8) unique? In fact, we have imposed the continuity boundary condition across $\Gamma_{a}$,

$$
\lim _{\mathbf{r} \rightarrow \mathbf{s}} \phi(\mathbf{r})=\left.\phi(\mathbf{s})\right|_{\mathbf{s} \in \partial \Omega_{a}} .
$$

This automatically removes the extra degree of freedom since any extra term $\phi^{k}(\mathbf{s})$ makes the field discontinuous when taking the limit to the boundary. Physically, if we are using multipole sources located inside the cloaking or illusion devices, the continuity boundary conditions must be satisfied.

However, as we know from Eq. (A12), the extra term $\phi^{k}$ does not influence the active fields outside the cloaking device. Therefore, instead of imposing the boundary continuity conditions, one can first find solutions of the form $\left\{\widetilde{\phi}_{a}, 0\right\}$ or $\left\{0, \partial_{\mathbf{n}} \widetilde{\phi}_{a}\right\}$ since both $\phi$ and $\partial_{\mathbf{n}} \phi$ can independently provide the required active sources "mathematically." In fact, physically, $\left\{\widetilde{\phi}_{a}, 0\right\}$ corresponds to placing dipoles on the boundaries and $\left\{0, \partial_{\mathbf{n}} \widetilde{\phi}_{a}\right\}$ corresponds to placing monopoles on the boundaries [see Eqs. (A2) and (A4)]. It is seen that one can use only the monopoles or only the dipoles placed on the
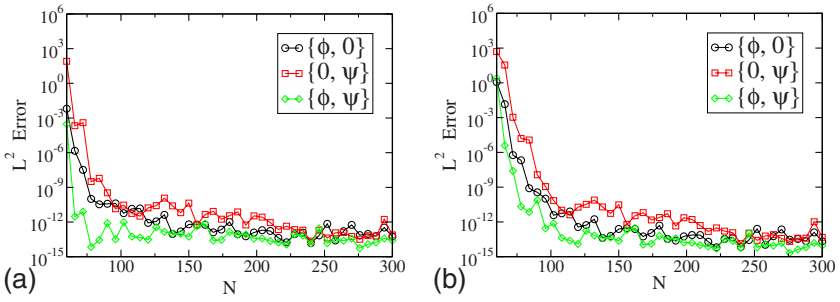

FIG. 8. (Color online) Errors on (a) $\Gamma_{c}: r=2.0$ and $\Gamma_{b}: r=20.0$, The wavelength $\lambda=10.0$. The errors are measured with the $L^{2}$ norm, defined in Eq. (13).

boundaries to obtain the required fields. For the $\left\{\widetilde{\phi}_{a}, 0\right\}$ type of solution, the active fields can be expressed as

$$
\phi^{\text {active }}(\mathbf{r})=-\int_{\partial \Omega_{a}} g(\mathbf{s}, \mathbf{r}) \partial_{\mathbf{n}} \widetilde{\phi}_{a}(\mathbf{s}) d s, \quad \mathbf{r} \in R^{2}-\Omega .
$$

Under this circumstance, the conditions for cloaking that the active fields should satisfy are

$$
-\int_{\partial \Omega_{a}} g(\mathbf{s}, \mathbf{r}) \partial_{\mathbf{n}} \widetilde{\phi}_{a}(\mathbf{s}) d s=\left\{\begin{aligned}
-\phi^{\text {inc }}(\mathbf{r}) & \text { for } \mathbf{r} \in \Gamma_{c}, \\
0 & \text { for } \mathbf{r} \in \Gamma_{b} .
\end{aligned}\right.
$$

One can also find the corresponding multipole sources, with the following strength coefficients:

$$
a_{m, j}=-\frac{i}{4} \oint_{\partial \Omega_{a_{j}}} d s e^{-i m \theta^{\prime}} J_{m}\left(k\left|\mathbf{s}-\mathbf{r}_{c_{j}}\right|\right) \partial_{\mathbf{n}} \widetilde{\phi}(\mathbf{s}) .
$$

The set of $a_{m, j}$ should be the same with that obtained from the first method in Eq. (A11). Please note that, in this case, we do not impose the boundary continuity conditions, therefore, if one takes the following limit from outside the cloaking device:

$$
\begin{gathered}
\left.\phi^{\text {phys. }}(\mathbf{s})\right|_{\mathbf{s} \in \partial \Omega} \doteq \lim _{\mathbf{r} \rightarrow \mathbf{s}} \phi(\mathbf{r}), \\
\left.\partial_{\mathbf{n}} \phi^{\text {phys. }}(\mathbf{s})\right|_{\mathbf{s} \in \partial \Omega} \doteq \lim _{\mathbf{r} \rightarrow \mathbf{s}} \partial_{\mathbf{n}} \phi(\mathbf{r}),
\end{gathered}
$$

generally, $\phi^{\text {phys. }}(\mathbf{s})$ is different with $\widetilde{\phi}_{a}(\mathbf{s})$. However, in principle, the fields $\left\{\phi^{\text {phys. }}, \partial_{\mathbf{n}} \phi^{\text {phys. }}\right\}$ should be the same with the $\left\{\phi_{a}, \partial_{\mathbf{n}} \phi_{a}\right\}$ acquired by imposing the boundary continuity conditions if the number of sample points $N$ is large. This method can reduce the dimension of the matrix by half. We present the numerical simulation results of different formalisms in Appendix B.

\section{APPENDIX B: CONVERGENCE DISCUSSION}

The numerical solution of Eq. (8) only ensures that Eq. (7) is satisfied for a finite number of points. Here, we consider the convergence of the solution as the number of sampling point increases by calculating the errors on the two circles $\Gamma_{c}$ and $\Gamma_{b}$. The errors are measured as Eq. (13) in the 

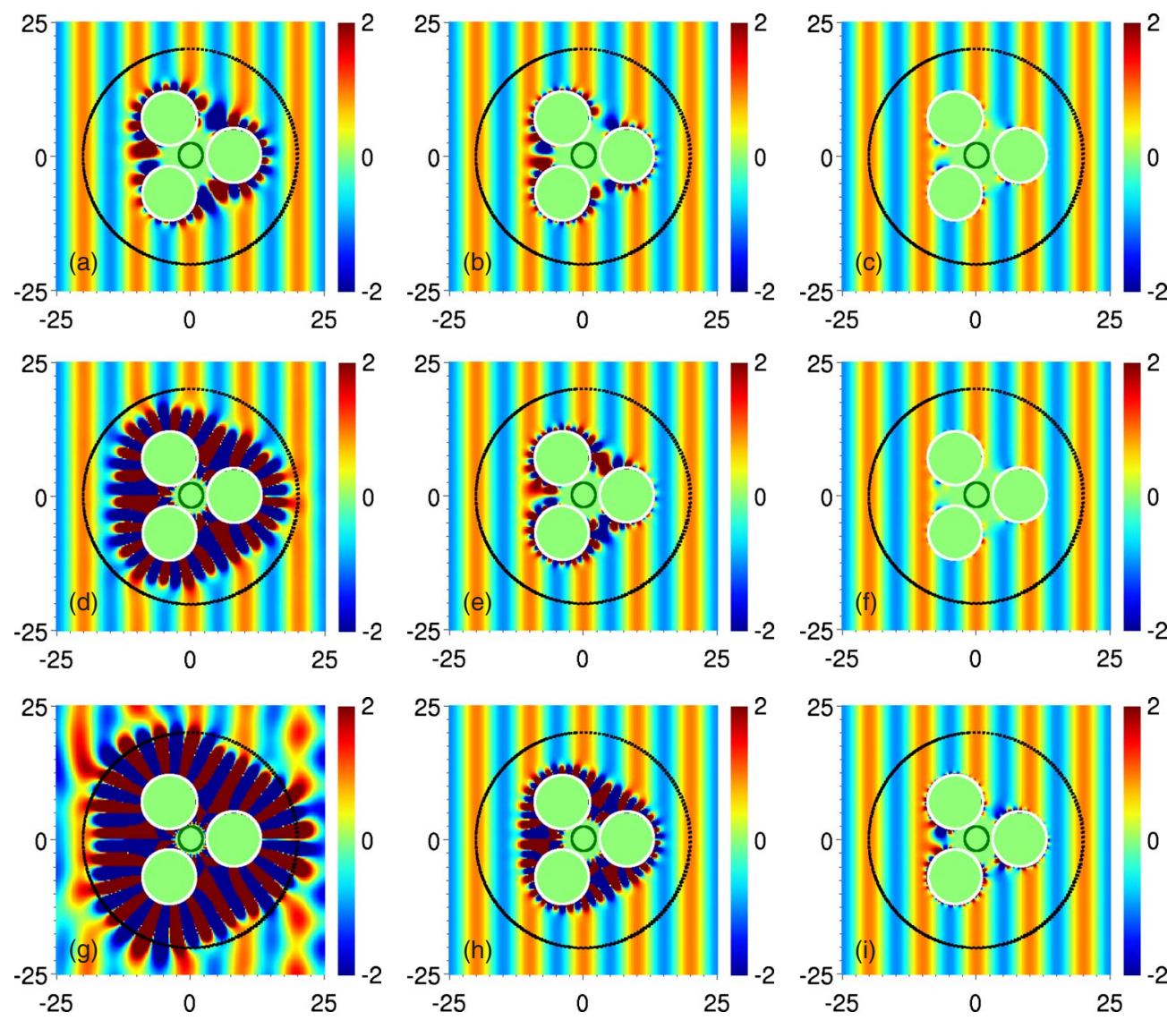

FIG. 9. (Color online) Field patterns of different schemes with different $N(N=66,90,240$ for left, middle, and right panels, respectively): $\Gamma_{c}=2.0$ and $\Gamma_{b}=20.0, \lambda=10.0$. (a), (b) and (c) are for $\phi_{a} \partial_{\mathbf{n}} \phi_{a}$ scheme; (d), (e) and (f) are for $\widetilde{\phi}_{a}, 0$ scheme; (g), (h) and (i) are for $0, \partial_{\mathbf{n}} \widetilde{\phi}_{a}$ scheme.

text. As shown in Fig. 8, the error decreases very quickly as $N$ increases (note that the vertical axis is in log scale). When $N$ is greater than 300 (this number, however, depends on the wavelength and the geometry of the cloaking device), the total error is of $\sim 10^{-14}$. This indicates that the field are essentially the same as the incoming wave outside the circle of $r=20.0$ and nearly zero inside the quiet zone inside the circle of $r=2.0$, which confirms that for all the practical purposes, objects concealed inside the quiet zone are undetectable out- side of $\Gamma_{b}$. To get a better picture of the performance of the cloaking as $N$ increases, we provide some representative field patterns of the three schemes in Fig. 9. As can be clearly seen, when $N$ is not large enough, there will be a lot of evident "tails" reaching out from the boundary of the cloaking device and the field values are large while for increasing $N$, the tails become less conspicuous. Meanwhile, the field patterns of the three different schemes look indistinguishable with each other as $N$ increases.
${ }^{1}$ M. Kerker, J. Opt. Soc. Am. 65, 376 (1975).

${ }^{2}$ A. Alù and N. Engheta, Phys. Rev. E 72, 016623 (2005).

${ }^{3}$ L. S. Dolin, Izv. Vyssh. Uchebn. Zaved., Radiofiz. 4, 964 (1961).

${ }^{4}$ U. Leonhardt, Science 312, 1777 (2006).

${ }^{5}$ U. Leonhardt and T. G. Philbin, New J. Phys. 8, 247 (2006).

${ }^{6}$ J. B. Pendry, D. Schurig, and D. R. Smith, Science 312, 1780 (2006).

${ }^{7}$ D. Schurig, J. J. Mock, B. J. Justice, S. A. Cummer, J. B. Pendry, A. F. Starr, and D. R. Smith, Science 314, 977 (2006).

${ }^{8}$ A. Greenleaf, M. Lassas, and G. Uhlmann, Math. Res. Lett. 10, 685 (2003).

${ }^{9}$ A. Greenleaf, M. Lassas, and G. Uhlmann, Physiol. Meas. 24, 413 (2003).
${ }^{10}$ J. Li and J. B. Pendry, Phys. Rev. Lett. 101, 203901 (2008).

${ }^{11}$ J. B. Pendry, Nature (London) 460, 579 (2009).

${ }^{12}$ A. V. Kildishev, W. Cai, U. K. Chettiar, and V. M. Shalaev, New J. Phys. 10, 115029 (2008).

${ }^{13}$ M. Farhat, S. Guenneau, and S. Enoch, Phys. Rev. Lett. 103, 024301 (2009).

${ }^{14}$ J. Valentine, J. Li, T. Zentgraf, G. Barta, and X. Zhang, Nat. Mater. 8, 568 (2009).

${ }^{15}$ Y. Lai, H. Chen, Z.-Q. Zhang, and C. T. Chan, Phys. Rev. Lett. 102, 093901 (2009).

${ }^{16}$ Y. Lai, J. Ng, H. Y. Chen, D. Z. Han, J. J. Xiao, Z.-Q. Zhang, and C. T. Chan, Phys. Rev. Lett. 102, 253902 (2009).

${ }^{17}$ D. A. B. Miller, Opt. Express 14, 12457 (2006). 
${ }^{18}$ F. Guevara Vasquez, G. W. Milton, and D. Onofrei, Phys. Rev. Lett. 103, 073901 (2009).

${ }^{19}$ G. W. Milton and N. A. P. Nicorovici, Proc. R. Soc. London, Ser. A 462, 3027 (2006).

${ }^{20}$ N. A. Nicorovici, G. W. Milton, R. C. McPhedran, and L. C. Botten, Opt. Express 15, 6314 (2007).

${ }^{21}$ F. Guevara Vasquez, G. W. Milton, and D. Onofrei, Opt. Express 17, 14800 (2009).
${ }^{22}$ J. J. Xiao and C. T. Chan, J. Opt. Soc. Am. B 25, 1553 (2008).

${ }^{23}$ W. C. Chew, J.-M. Jin, E. Michielssen, and J. Song, Fast and Efficient Algorithms in Computational Electromagnetics (Artech, Boston, 2001).

${ }^{24}$ J. A. Kong, Electromagnetic Wave Theory (EMW, Cambridge, Massachusetts, 2005).

${ }^{25}$ J. Wiersig, J. Opt. A, Pure Appl. Opt. 5, 53 (2003). 